\title{
UN CASO DI SPIONAGGIO DEL XVIII SECOLO: IL VIAGGIO-RICOGNIZIONE A POLA DI UN GRUPPO DI FUNZIONARI DELL'IMPERATORE ASBURGICO. APPUNTI DA UNA RELAZIONE ANONIMA AL CONTE E PROVVEDITORE DI POLA DEL 12 APRILE 1788
}

\author{
Sandro Cergna
}

\section{RIASSUNTO}

Nel lavoro si presenta il viaggio compiuto da un gruppo di funzionari della corte imperiale di Giuseppe II, nel 1788, da Fiume, attraverso alcune località della costa orientale dell'Istria, fino a Pola. Facendo riferimento al documento originale, una relazione anonima trasmessa al Conte e Provveditore della città istriana, custodito nell'archivio della biblioteca Universitaria di Pola, si riporta il tragitto della comitiva austro-ungarica e, più dettagliatamente, l'incontro del gruppo con l'anonimo informatore dell'allora Reggitore della città istriana, Angelo Maria Orio, neanch'egli nominato nella relazione. Il documento, accanto all'interesse odeporicoculturale dello scritto, rileva pure interessanti notizie di carattere storicopolitico, quali le nascoste motivazioni che hanno portato gli illustri personaggi a ricercare quante più informazioni sulla città, sul porto e sulle vie di comunicazione di Pola; il loro interessamento in merito alle politiche economiche e di difesa della Serenissima in queste terre, a meno di dieci anni dalla caduta della stessa. Illustra, inoltre, la situazione politica dell'Istria e delle realtà statali contermini del tempo.

Parole chiave: Istria, Pola, viaggio, spionaggio, Venezia, Austria, Ungheria 
Fin dai primi secoli della sua storia, la Repubblica di Venezia creò un complesso ed efficiente sistema spionistico per vigilare sulla sicurezza interna ed esterna, controllato, a partire dagli inizi del XIV secolo, dal Consiglio dei Dieci. L'apparato repressivo statale poteva inoltre contare su un'ampia rete di informatori, confidenti, spie e spioni locali, che riferivano all'autorità competente quanto veniva ad essi richiesto di indagare. Da un manoscritto ritrovato nell'archivio della Biblioteca universitaria di Pola ${ }^{[1]}$, veniamo a sapere che anche la città istriana, nell'ultimo decennio di vita della Serenissima, fu teatro di un episodio spionistico, documentato da un anonimo informatore del Conte Provveditore della città istriana, Angelo Maria Orio ${ }^{[2]}$, dal quale il servizio era stato commissionato.

Fig. 1. Il fascicolo contenente il documento.

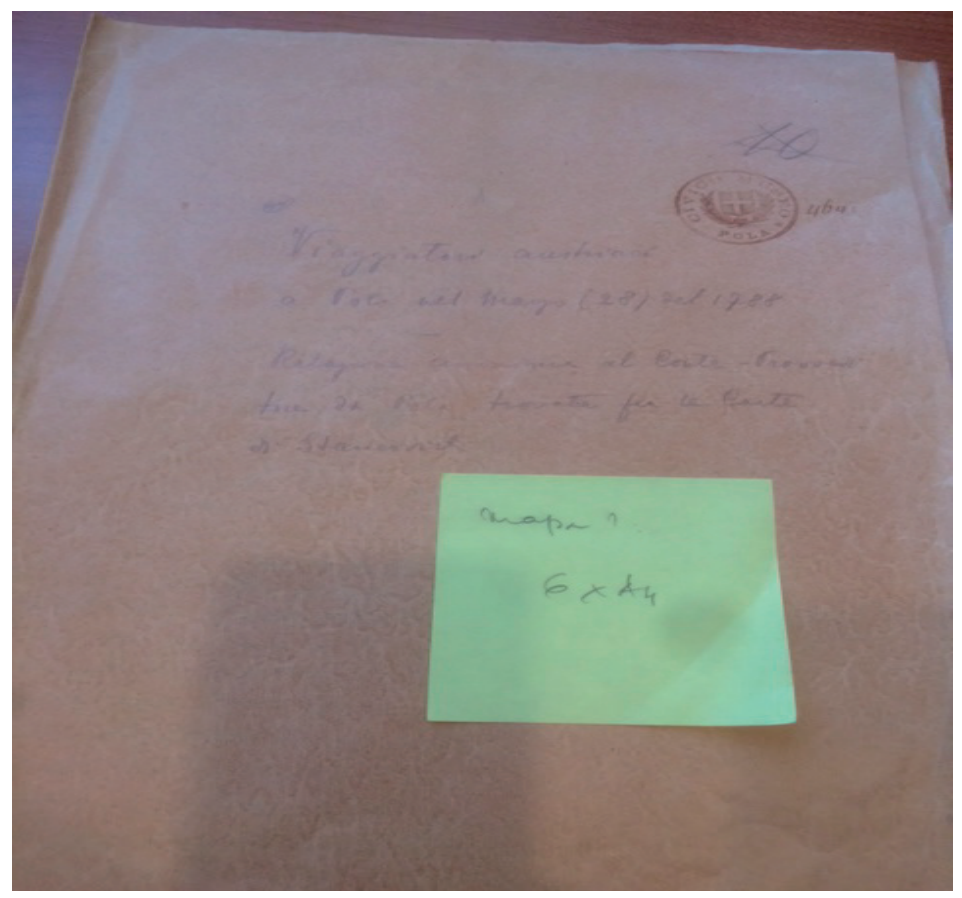

[1] Scatola VIII, fasc. 1, consta di 3 pagine, di cm 29x20. Il titolo sul frontespizio del documento recita: "Viaggiatori austriaci a Pola nel marzo (28) del 1788. Relazione anonima al Conte Provveditore di Pola trovata fra le Carte di Stancovich".

[2] Anzolo Maria Orio fu Provveditore di Pola dal 20 marzo 1788 al 20 luglio 1789. Un autografo originale dell'Orio compare in un documento datato Pola, 15 aprile 1789; cfr. Silvio Mitis, Documenti per la storia di Pola, Atti e memorie della Società istriana di archeologia e storia patria, vol. XXVII, anno XXVIII, Parenzo 1911, p. 3. Nel manoscritto dell'archivio di Pola non compare il nome del Provveditore. 
Il documento rappresenta il resoconto del viaggio che un gruppo di alti funzionari del regno d'Ungheria compì tra il 26 e il 29 marzo 1788 lungo il tragitto da Fiume a Pisino. Partendo dal capoluogo quarnerino, la comitiva visitò alcune cittadine della costa orientale dell'Istria, per giungere, infine, a Pola e proseguire successivamente, attraverso Dignano e Gimino, verso la capitale della Contea d'Istria - Pisino. Non si trattò, però, di un viaggio di diletto, quanto bensì, di un'escursione in territorio veneziano, precedentemente pianificata, con il preciso compito di portare a termine un atto di spionaggio tecnologico-industriale e militare, consistente nell'individuare quale potesse essere l'ubicazione più adatta alla costruzione di un futuro porto, nonché prevedere la più agevole via di collegamento tra esso e la città di Pisino, e da lì con Vienna.

È quanto emerge dalla relazione anonima, trovata, come è riportato sul fascicolo che la contiene, "fra le Carte di Stancovich", e custodita nell'archivio della Biblioteca universitaria polese. Nel presente lavoro si analizza il viaggio in missione d'intelligence della comitiva ungherese e l'incontro tra i protagonisti di quel tragitto e un anonimo informatore del Provveditore veneziano di Pola, nel marzo del 1788, solo nove anni prima della caduta della Serenissima.

Degli otto "Austriaci incogniti viaggiatori" che, per "fortuito incontro", l'anonimo agente veneziano incontra il 28 marzo 1788 nell'anfiteatro di Pola, si riporta il cognome solo di due personaggi: Zapari, ossia il conte Jànos Pétar Szàpary ${ }^{[3]}$, Governatore di Fiume, e Palfij, ovvero il conte Palffy, "nipote del gran Can:re del Regno di Ungaria"[4]. Gli altri sei funzionari sono: un barone ungherese, "il p. ${ }^{\mathrm{mo}}$ Cap: Circolare della Croazia, un graduato uffiziale ingegnere senza alcun vestito, o altra insegna militare, un Reg: Ces: Segretario di Commissariato, un [...], ed il

[3] Il conte Jànos Pétar Szàpary (1757-1815) ricoprì la carica di Governatore di Fiume dal 1788 al 1791. Cfr. W. KLINGER, La guerra di successione spagnola e le origini dell'emporio di Fiume (1701 - 1779), Atti, Centro di Ricerche Storiche, Rovigno, vol. XLIV, 2014, p. 83.

[4] Come si legge nella relazione di Daniele Dolfin, ambasciatore presso la corte di Vienna e presentata al Senato della Repubblica di Venezia nel 1793, Gran Cancelliere del Regno d'Ungheria fu, in quegli anni, il conte Karl Palffy (1735-1816); scrive Dolfin: "Quando nella conferenza di Stato si trattano affari risguardanti il regno di Boemia, o quello d’Ungheria, sogliono rispettivamente chiamarsi i due Gran Cancellieri; cioè, il Conte Kollowrat per il primo, e il Conte Carlo Palffy per il secondo. Questi due Ministri sono molto intelligenti e pratici per la direzione interna dei summenzionati Regni, ed essendovi influentissimi per aderenze e per parentele, sono riputati, considerati, e non poco accarezzati alla Corte", in Fontes Rerum Austriacarum. Oesterreichische Geschichts-Quellen. Zweite Abtheilung. Diplomataria. XXII Band. Die Relationen der Botschafter Venedigs über Österreich im Achtzenten Jahrhundert, Wien 1863, p. 351. Più difficile risulta identificare chi fosse il Palffy nipote che partecipò al viaggio in Istria. Uno tra i possibili nipoti di Karl potrebbe essere Johann Palffy (1744-1794), come pure Ferdinand Palffy (1774-1840), oppure Joseph Palffy (1764-1827). 
Mastro di Posta di quella Città", al cui seguito partecipava un folto numero di servitori con bagagli e cavalli.

Il viaggio in Istria, però, era stato anticipato da una precedente esplorazione di due giorni lungo il litorale fiumano, da dove, riporta l'informatore, un cordone militare di duemila uomini si stendeva fino al "veneto confine presso il castello di Fianona", e durante la quale gli ospiti ungheresi furono accompagnati da un Generale di Stato maggiore di Carlstadt [Karlovac], "capitale della Croazia".

Per raggiungere la penisola istriana, i membri della comitiva partono il 26 marzo dal porto franco di Fiume ${ }^{[5]}$ su una "felucca imperiale", a bordo della quale arrivano al Castello di Fianona, per poi recarsi, "con pronte vetture", ad Albona, dove trascorreranno la notte.

Il giorno seguente alcuni membri del gruppo si recano a visitare il vicino Porto Rabaz e, successivamente, nuovamente riunitisi e attraversato il canale dell'Arsa, riprendono il tragitto raggiungendo in serata il piccolo villaggio di Villa Carnizza. La mattina successiva, 28 marzo, i viaggiatori si recano a visitare il Porto Lungo [Duga uvala] da dove, osserva l'informatore, anziché intraprendere la strada più agibile attraverso Villa Marzana, si avviano a visitare il Porto Badò di Villa Altura, per poi da lì condursi verso Pola. Nell'anfiteatro della città istriana, dove li ritroviamo lo stesso 28 marzo, avviene l'incontro tra l'informatore e i visitatori che, annota quello, muniti di cannocchiali "un poco più grandi di quelli si usano ne Teatri", stavano intenti ad osservare con attenzione il porto antistante l'antico monumento, esternando spesso esclamazioni di ammirazione in tedesco. Mosso dal dubbio che gli stranieri non avessero còlto la magnificenza e la grandezza del vetusto teatro, l'autore descrive la modalità del singolare incontro, riconoscendo al contempo l'erroneità della sua iniziale supposizione:

Non so se prima avessero dirette le loro attenzioni a quel superbo edifizio, dirò bensì, che riuniti si diressero verso la città, quando un ragionevole dubbio, che non avessero compresi la forma interna, l'oggetto, e l'uso di quel anfiteatro, mi stimolò di presentarmi alli a me allora ignoti viaggiatori, cui fatto un civile uffizio, mi offersi di dargliene i lumi, e fargliene la descrizione. Resomi subito seco loro nell'arena col miglior dittaglio mi fu

[5] Fiume fu porto franco dal 1719 al 1892. 
possibile li posi al fatto di ciò [...].

Ma è proprio mentre illustra al gruppo la storia della prestigiosa costruzione che l'improvvisato cicerone si avvede di come l'interesse degli ospiti non sia rivolto verso l'anfiteatro e le vicende storiche che l'hanno accompagnato, quanto, invece, all'insenatura del porto che si apriva di fronte all'arena, e di cui prendevano scrupolosi appunti e abbozzi:

A descrizione avvanzata mi avvidi che quell'illustre antico monumento ressiduo della Romana grandezza non interessava gran fatto li oggetti della loro curiosità [...]. Dirò essermi avveduto che l'ufficiale ingegnere tratto tratto rivolto ad altra parte andava sopra una carta non so se scrivendo, o facendo linee. Ora l'uno ora l'altro della brigata anziché interessarsi nelle notizie di queste antichità le cercavano piuttosto sopra il Porto.

Come emerge dal seguito della relazione, furono diversi i momenti che gradualmente portarono a confermare nel Nostro il sospetto che il motivo dell'arrivo degli alti personaggi a Pola fosse principalmente quello di esaminare e prendere nota dell'ubicazione e delle caratteristiche geostrategiche del porto di Pola: oltre all'incontro nell'anfiteatro, egli riuscì a carpire importanti informazioni al Mastro di Posta, con il quale si accompagnò per un tratto di strada da Pola a Dignano, ed ebbe ancora occasione di conversare con i membri della comitiva la sera stessa, sempre a Dignano. Non meno rilevanti, inoltre, per confermare i suoi sospetti sulle reali motivazioni del viaggio di quei funzionari del Regno d'Ungheria a Pola, furono pure le informazioni del giovane "villano accorto e parlatore", che fece da guida agli illustri ospiti accompagnandoli, la sera del 28 marzo, da Dignano a Gimino, "primo Castello del limitrofo Austriaco confine" da dove, recatisi il mattino seguente a Pisino, lasciarono l'Istria.

[...] e finalmente le notizie ritratte dopo la loro partenza fatta la mattina dei 29 da Pisino, ossia Mittelburgh luogo principale dell'interna Istria austriaca mi posero al fatto del grado, e Carattere di quei distinti soggetti, e mi confermarono nel sospetto, che li oggetti primari del loro viaggio sieno stati di rilevare l'ampiezza, la profondità, capacità, ingresso, uscita, e sicurezza di questo Porto. 
Accanto all'interesse per il rilevamento dei dati topografici del porto, i funzionari ungheresi cercavano pure di raccogliere informazioni su un altro importante elemento geostrategico del territorio: le vie di comunicazione e, in particolare, interessava loro sapere se vi fosse una strada romana che da Pola conducesse in Germania. Dalle insistite domande dei forestieri, l'agente veneziano giunge così a dedurre che l'imperatore stesso avesse disposto la costruzione di un nuovo collegamento viario tra il futuro porto, da realizzare in una tra le insenature visitate, e la già esistente strada che da Pisino proseguiva a nord verso il Monte Maggiore. Riportando, poi, quanto riferitogli dalla giovane guida in merito ai discorsi di questa con il conte Szàpary - non escludendo però il dubbio sulle rivelazioni della stessa guida -, l'informatore desume che l'eventuale futuro collegamento stradale sarebbe stato costruito per arrivare proprio fino alla baia di Pola.

Fui per parte mia riservato, ed inconcludente, e vi fui altresì quando chiesero, se gli antichi Romani avessero strada, che da questa Città di Pola andasse in Germania [...]. Fu allora che destramente or da l'uno or dall'altro separatamente cercai comprendere l'oggetto di S. M. R. I. in formare la nuova strada, che superba e dispendiosa si continua anche in presente, e dal Monte Maggiore trenta miglia c: ${ }^{\mathrm{a}}$ lontano da qui si dirigge verso Pisino. Benché variassero le risposte, e di alcuni anche oscure, pure v'è motivo di sospettare, che si contempli la sua direzione fino ad un Porto di qta Provincia. [...] potrei dire, che S. E. Co. Zapari si espresse, che detta strada terminerà a questo Porto di Pola.

A conclusione del verbale, l'anonimo informatore tocca pure l'argomento geopolitico e militare. soffermandosi su due domande del conte Szàpary in merito all'eventuale disposizione di posti militari lungo le coste della Serenissima e se, qualora ciò avvenisse, tali milizie sarebbero intervenute a difesa delle navi veneziane. La risposta dell'agente veneziano, a pochi anni prima della caduta di Venezia, è improntata ad un atteggiamento di fiduciosa sicurezza: un Principe in pace, lontano dalla guerra, non ha bisogno di tali precauzioni giacché "[...] una rispettabile squadra comandata dal famoso S. E. [...] Emo era nell'acque del Levante”.

Tale graduale acquisizione delle reali motivazioni che mossero quei personaggi a visitare l'Istria, accanto agli altri macrosegni che possiamo 
individuare nel breve testo (i personaggi, il viaggio, l'incontro) trovano corrispondenze formali ed ideologiche con la letteratura di spionaggio. E vi è un ulteriore elemento che ci autorizza a un tale raffronto: la descrizione psicologica dell'autore-protagonista. Scaltro e sospettoso, nella sua autorappresentazione l'autore dello scritto si avvicina all'investigatore Auguste Dupin di Poe: alle domande degli illustri ospiti non si pronuncia apertamente, non dà risposte precise, rimane nel vago, cercando, al contempo, di carpire informazioni utili dai suoi interlocutori dandoci, così, in fugaci ma illuminanti schizzi, il ritratto dell'agente di intelligence.

Il verbale dell'incontro tra il gruppo di funzionari austroungarici e il confidente del Provveditore Anzolo Maria Orio, pertanto, oltre ad essere un interessante documento di spionaggio militare e industrialecommerciale e di controspionaggio tra due grandi potenze: l'ormai morente Repubblica di Venezia e la vitale monarchia di Giuseppe II, oscilla al contempo tra la letteratura odeporica e il racconto di spionaggio. Lagente veneziano comprende molto presto gli intenti dei personaggi che ha di fronte e le motivazioni della loro visita a Pola; con acutezza e puntualità sa cogliere le sfumature dei discorsi dei suoi interlocutori, come quando registra, tradotta, la ripetuta curiosa esclamazione: "Sacrament! Qsto Porto in mano del nostro Sovrano ${ }^{[6]}$ sarebbe in pochi anni un altro Amsterdam", apportando così vividezza ed efficacia narrativa ad un testo burocratico e di ordinaria amministrazione, qual è un verbale, nonché informandoci su alcuni aspetti della situazione politica dell'Istria e degli Stati contermini dell'epoca.

[6] Giuseppe II (1741-1790), alla morte del padre Francesco I nel 1765, fu coreggente con la madre Maria Teresa dell'ampio dominio degli Asburgo e, dal 1780, imperatore del Sacro Romano Impero. Rappresentante del "dispotismo illuminato", introdusse numerose riforme in ambito amministrativo, giuridico ed ecclesiale. 


\section{BIBLIOGRAFIA}

Fontes Rerum Austriacarum (1863). Oesterreichische Geschichts-Quellen. Zweite Abtheilung. Diplomataria. XXII Band. Die Relationen der Botschafter Venedigs über Österreich im Achtzenten Jahrhundert, Wien.

Klinger, William (2014). La guerra di successione spagnola e le origini dell'emporio di Fiume (1701 - 1779), Atti, Centro di Ricerche Storiche, Rovigno, vol. XLIV.

Mitis, Silvio (1911). Documenti per la storia di Pola, Atti e memorie della Società istriana di archeologia e storia patria, vol. XXVII, anno XXVIII, Parenzo.

\section{A CASE OF ESPIONAGE FROM THE 18TH CENTURY: A RECONNAISSANCE TRIP TO POLA MADE BY A GROUP OF INTELLIGENCE OFFICERS OF THE HABSBURG EMPEROR. NOTES FROM THE ANONYMOUS REPORT TO THE GOVERNOR OF POLA DATED 12 APRIL 1788}

This paper gives an account of a trip made by a group of officials of Emperor Joseph II from 1788, from Rijeka to Pula, while passing through several towns and villages of the eastern coast of Istria. The original document, that is, an anonymous report addressed to the Governor of the Istrian city, has been preserved in the archives of the University Library in Pula. It describes the journey of the Austro-Hungarian group and, in more detail, their encounter with an anonymous intelligence officer of the then prefect of the Istrian city, Angelo Maria Orio, who was also not named in the report. This document, significant as a travelogue as well as in terms of culture, also contains interesting historical and political information, such as the hidden reasons why these famous characters sought as much information as possible about Pula, its port and transport routes; their interest in economic policy and defence of the Venetian Republic in this area not even ten years before its fall. It also describes the political situation in Istria and the state border situation at the time.

Keywords: Istria, Pola, trip, secret service, Venezia, Austria, Hungary 